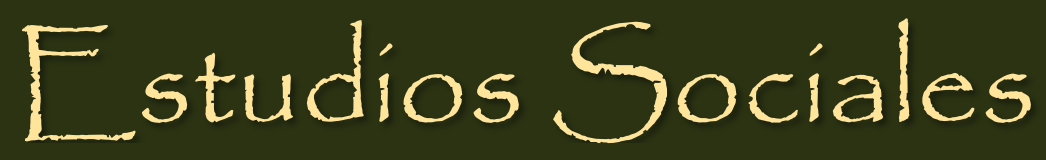

Revista de Alimentación Contemporánea y Desarrollo Regional

Volumen 31, Número 58. Julio - Diciembre 2021

Revista Electrónica. ISSN: 2395-9169

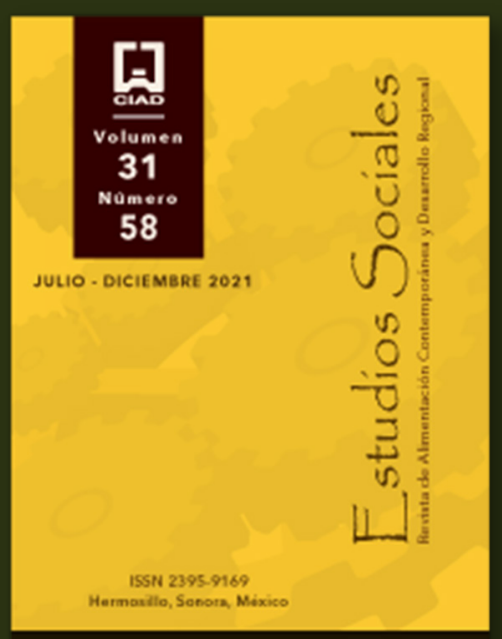

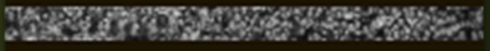

Estado de la información del consumo en México de antioxidantes sintéticos en alimentos ultra-procesados, basados en los productos de la canasta básica

State of the information on the consumption in Mexico of synthetic antioxidants in ultra-processed foods, based on the products of the basic basket

DOI: https://doi.org/10.24836/es.v31i58.1143

e211143

Jocelyn Astrid Carbajal-Sánchez*

https://orcid.org/0000-0002-6807-1388

Ninfa Ramírez-Durán*

https://orcid.org/0000-0003-3108-895X

Marcela Gamboa-Angulo**

https://orcid.org/0000-0002-0618-0335

Pablo Antonio Moreno-Pérez*

https://orcid.org/0000-0002-5755-8959

Fecha de recepción: 23 de junio de 2021.

Fecha de envío a evaluación: 27 de agosto de 2021.

Fecha de aceptación: 10 de septiembre de 2021.

*Universidad Autónoma del Estado de México.

${ }^{* *}$ Centro de Investigación Científica de Yucatán, México.

Autor para correspondencia: Pablo Antonio Moreno-Pérez.

Av. Paseo Tollocan, C. Jesús Carranza, Moderna de la Cruz 50180 Toluca de Lerdo, México.

Tel. 729-249-2946

Dirección: saieto@hotmail.com

Centro de Investigación en Alimentación y Desarrollo, A. C.

Hermosillo, Sonora, México. 


\section{ESTADO DE LA INFORMACIÓN DEL CONSUMO EN MÉXICO DE ANTIOXIDANTES SINTÉTICOS \\ EN ALIMENTOS ULTRA-PROCESADOS, BASADOS EN LOS PRODUCTOS DE LA CANASTA BÁSICA \\ Carbajal-SÁnchez, Ramírez-Durán, Gamboa-Angulo, Moreno-PÉrez}

\section{Resumen / Abstract}

Objetivo: Dar a conocer el estado de la información del consumo de antioxidantes sintéticos en alimentos ultraprocesados en México con base en los productos de la canasta básica. Metodología: Se seleccionaron los alimentos disponibles, envasados o enlatados, que comprende la canasta básica mexicana de tiendas de conveniencia y misceláneas representativas de los 125 municipios del Estado de México. Se registró la información del contenido y concentración de los antioxidantes sintéticos (AS) butilhidroxianisol (BHA), butilhidroxitolueno (BHT) y terbutilhidroquinona (TBHQ), posteriormente los alimentos se clasificaron bajo el sistema NOVA. Resultados: Se encontraron 53 productos alimenticios derivados de la canasta básica mexicana que son comercializados envasados o enlatados, el $71 \%$ menciona el tipo de antioxidante utilizado y solo el $18 \%$ la concentración. De acuerdo con la clasificación NOVA, más del 73\% de los alimentos pertenecen a la clasificación cuatro de productos ultraprocesados, de los cuales solo once reportan el antioxidante sintético utilizado, ninguno menciona la concentración. Limitaciones: Se carece de información del contenido y concentración del antioxidante utilizado en la etiqueta de información nutricional. Conclusiones: Solo el $18 \%$ de los productos analizados informa la concentración del antioxidante sintético utilizado, consecuentemente la ingestión diaria admisible puede ser subestimada. Es necesario realizar más investigaciones sobre la exposición dietética a AS en los mexicanos.

Palabras clave: alimentación contemporánea; alimentos; aditivos; ultraprocesados; antioxidantes; sintéticos.
Objective: To present the status of the information on the consumption of synthetic antioxidants in ultra-processed foods in Mexico, depending on the products of the basic basket. Methodology: The foods available packaged or canned, that comprise the basic Mexican basket, from convenience stores and miscellaneous representative of the 125 municipalities of the state of Mexico were selected. The information on the content and concentration of synthetic antioxidants was recorded (AS) butylhydroxyanisole (BHA), butylhydroxytoluene (BHT) and tert-butylhydroquinone (TBHQ), subsequently the foods were classified under the NOVA system. Results: 53 food products derived from the basic Mexican basket were found that are marketed packaged or canned, $71 \%$ mentioned the type of antioxidant used and only $18 \%$ the concentration. According to the NOVA classification, more than $73 \%$ of the foods belong to classification 4 of ultra-processed products, of which only 11 report the synthetic antioxidant used, none mention the concentration. Limitations: There is no information on the content and concentration of the antioxidant used on the Nutrition Facts label. Conclusions: Only $18 \%$ of the analyzed products report the concentration of the synthetic antioxidant used, consequently the acceptable daily intake can be underestimated. More research is needed on dietary exposure to AS in Mexicans.
Key words: contemporary food; food; additives; ultra-processed; antioxidants; synthetics. 


\section{Introducción}

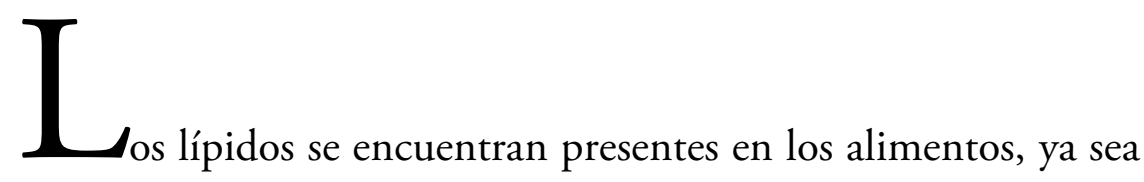
de forma natural o agregados durante su procesamiento (Shahidi y Abad, 2018), sin embargo, la oxidación de lípidos o rancidez oxidativa provoca cambios organolépticos y físico-químicos indeseables en los alimentos, como cambios en el color, sabor y textura, afectando su calidad y seguridad (Atta, Mohamed y Abdelgawad, 2017; Domínguez et al., 2019; Lourenço, Moldáo-Martins y Alves, 2019). Para prevenir esta problemática, se adicionan a los alimentos procesados los antioxidantes definidos como "cualquier sustancia que, cuando está presente en concentraciones bajas en comparación con las de un sustrato oxidable, retrasa o previene significativamente la oxidación de ese sustrato" (Halliwell, 1995), esto con la finalidad de retrasar la oxidación de las biomoléculas de los alimentos (Lourenço et al., 2019), disminuyendo el efecto perjudicial del estrés oxidativo (Salehi et al., 2018; Sugiharto, 2019). 
Los antioxidantes se pueden clasificar en naturales y sintéticos, los alimentos procesados o ultraprocesados generalmente contienen antioxidantes sintéticos (AS) adicionados, como el compuesto monofenólico que se compone de los isómeros 2terc-butil-4-hidroxianisol y 3-tercbutil-4-hidroxianisol (BHA), el 2,6-di-terc-butil4-metilfenol (BHT), que procede de la industria petrolera y el 2-terc-butil-1,4bencenodiol (TBHQ), que es un compuesto fenólico (Sun et al., 2019; Zhao, Zhao, Liu y Wang, 2020; Xu et al., 2021), todos comúnmente utilizados en la industria cosmética y alimentaria debido a su disponibilidad, estabilidad química y bajo costo (Shankar et al., 2020). México es uno de los mayores consumidores de productos ultraprocesados en América Latina (OPS y OMS, 2015), consecuentemente los AS son consumidos por toda la población mexicana, incluyendo personas que presentan algún padecimiento, adultos mayores y población infantil. A pesar de que la Secretaría de Salud regula los límites máximos de cada antioxidante en México, la mayoría de los productos envasados o enlatados disponibles en el mercado mexicano no especifican la concentración, esto puede repercutir en el desarrollo de enfermedades a largo plazo debido a una ingestión de AS mayor de a la recomendada, lo que puede resultar en carcinogenicidad, citotoxicidad, inducción de estrés oxidativo y efectos de alteración endocrina ( $\mathrm{Xu}$ et al., 2021), investigaciones recientes han informado la toxicidad causada por BHA, BHT y TBHQ (Tabla 1). 


\section{ESTUDIOS SOCIALES}

ISSN: 2395-9169

Tabla 1. Información de la toxicidad de los antioxidantes $B H T, B H A, T B H Q$

\begin{tabular}{|c|c|c|c|c|}
\hline Antioxidante & Evaluado en & Toxicidad & Concentración/ dosis & Referencia \\
\hline BHA & Embriones de Danio rerio & Cambios degenerativos y necrosis en el cerebro & $1,5,7,5$ y 10 ppm & (Baran et al., 2020) \\
\hline BHA & Larvas de Danio rerio & Disturbios en el metabolismo & $1,2 \mathrm{mg} / \mathrm{L}$ & $\begin{array}{l}\text { (Zhao, Xu, Yan, Ren, y } \\
\text { Zhang, 2020) }\end{array}$ \\
\hline BHA & Células de ratón Leydig y Sertoli & Induce disfunción testicular & $100 \mu \mathrm{M}$ & $\begin{array}{l}\text { (Ham, Lim, You, y Song, } \\
\text { 2020) }\end{array}$ \\
\hline BHA & Astrocitos humanos normales & $\begin{array}{l}\text { Neurotoxicidad al mejorar la acumulación de calcio } \\
\text { citosólico y el estrés del retículo endoplásmico }\end{array}$ & $100 \mu \mathrm{M}$ & $\begin{array}{l}\text { (Park, Lee, Lim, You, y Song, } \\
\text { 2019) }\end{array}$ \\
\hline BHA & $\begin{array}{l}\text { Ratones machos } \mathrm{C} 57 \mathrm{BL} / 6 \mathrm{~J} \text { de } 4 \text { semanas } \\
\text { de edad }\end{array}$ & $\begin{array}{l}\text { Obesógeno que puede afectar la acumulación de tejido } \\
\text { adiposo blanco y los lípidos plasmáticos }\end{array}$ & $\begin{array}{l}10 \mathrm{mg} / \mathrm{kg} \text { durante } 18 \\
\text { semanas }\end{array}$ & (Sun et al., 2019) \\
\hline BHA & Danio rerio & Inhibe la eclosión embrionaria & $>20 \mu \mathrm{M}$ & $\begin{array}{l}\text { (Yang, Sun, Wang, Zhou, y } \\
\text { Shi, 2018) }\end{array}$ \\
\hline BHA & $\begin{array}{l}\text { Ratas albinas Sprague-Dawley macho } \\
\text { adultas }\end{array}$ & Causa anormalidades en el ADN & $\begin{array}{l}0.7 \mathrm{mg} / \mathrm{kg} \text { de peso por } \\
60 \text { días }\end{array}$ & (Abo-EL-Sooud et al., 2018) \\
\hline BHA & $\begin{array}{l}\text { Línea celular de carcinoma de pulmón } \\
\text { A5 } 49 \text {. }\end{array}$ & Citotoxicidad & $0.3,0.4$, y $0.55 \mathrm{mM}$ & (Vandghanooni et al., 2013) \\
\hline BHT & Ratones Balb/c hembras & Acumulación en hígado y riñón & $200 \mathrm{mg} / \mathrm{kg}$ & (Zhang, Li, y Cui, 2020) \\
\hline BHT & Células de Ishikawa & Reprime la expresión basal de genes sensibles al estrógeno & $1,10,100$ y $1000 \mathrm{nM}$ & (Alofe et al., 2019) \\
\hline BHT & $\begin{array}{l}\text { Células de Leydig de ratón TM3 y células } \\
\text { de Sertoli de ratón TM4 }\end{array}$ & $\begin{array}{l}\text { Induce desequilibrio de la homeostasis del calcio y el estrés } \\
\text { del retículo endoplásmico que resulta en la muerte de las } \\
\text { células de Leydig }\end{array}$ & $100 \mu \mathrm{M}$ & $\begin{array}{l}\text { (Ham, Lim, Whang, y Song, } \\
\text { 2019) }\end{array}$ \\
\hline BHT & Larvas de Danio rerio & $\begin{array}{l}\text { Deformidades de embriones, disminución de la frecuencia } \\
\text { cardiaca, induce hiperactividad }\end{array}$ & 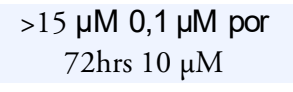 & (Liang et al., 2019) \\
\hline TBHQ & Embriones de Danio rerio & $\begin{array}{l}\text { Cambios degenerativos y necrosis en el cerebro, induce } \\
\text { apoptosis }\end{array}$ & $2.5,3.75$ y 5 ppm & (Baran et al., 2020) \\
\hline TBHQ & $\begin{array}{l}\text { Células endoteliales de la vena umbilical } \\
\text { humana }\end{array}$ & Estimula la citotoxicidad e induce apoptosis temprana & $60 \mu \mathrm{M}$ & $\begin{array}{l}\text { (Karimi, Ezzati, Dolatabadi, y } \\
\text { Dehghan, 2019) }\end{array}$ \\
\hline TBHQ & Danio rerio & Efecto letal en larvas & $>20 \mu \mathrm{M}$ & (Yang et al., 2018) \\
\hline TBHQ & $\begin{array}{l}\text { Línea celular de carcinoma de pulmón } \\
\text { A549 y células endoteliales de la vena } \\
\text { umbilical humana }\end{array}$ & Estimula apoptosis y carcinogenicidad & $5 \times 10^{-4} \mathrm{M}$ & $\begin{array}{l}\text { (Eskandani, Hamishehkar, } \\
\text { Ezzati, y Dolatabadi, 2014) }\end{array}$ \\
\hline
\end{tabular}

Fuente: elaboración propia. $\mathrm{BHA}=$ butilhidroxianisol. $\mathrm{BHT}=$ butilhidroxitolueno. $\mathrm{TBHQ}=$ terbutilhidroquinona. ppm= partes por millón 
A pesar de que los antioxidantes BHA, BHT y TBHQ son frecuentemente consumidos por la población mexicana, la mayoría de los productos envasados o enlatados carece de la información precisa de su contenido, esta característica favorece el desconocimiento a la exposición a estos aditivos alimentarios en México.

La canasta básica en México se refiere al conjunto de insumos, entre los que se incluyen los alimentos, indispensables para satisfacer las necesidades básicas de alimento de una familia a partir de su ingreso (Martínez, 2000), la mayor proporción de los alimentos que la comprenden, se encuentran en su origen natural, sin procesar, aunque también se encuentran disponibles envasados o enlatados, por lo que se pueden clasificar como alimentos o productos ultra procesados (PUP), que son formulaciones industriales, principalmente a base de sustancias extraídas de fuentes orgánicas (OPS, 2015; OMS, 2015).

Los PUP se encuentran dentro de la clasificación NOVA que clasifica a los alimentos y productos alimenticios de acuerdo con el alcance y la finalidad del procesamiento industrial al que se someten. Considera todos los métodos físicos, biológicos y químicos usados en el proceso de fabricación, incluido el uso de aditivos, la clasificación NOVA clasifica a los alimentos en cuatro grupos: NOVA 1: alimentos sin procesar o mínimamente procesados, NOVA 2: ingredientes culinarios procesados, NOVA 3: alimentos procesados (Productos industriales elaborados mediante la adición de sal, azúcar u otra sustancia), utilizando métodos de conservación como el envasado y embotellado, por último NOVA 4: Alimentos ultra-procesados (formulaciones de ingredientes, en su mayoría de uso industrial exclusivo, que resultan de una serie de procesos industriales) (OPS, 2015; OMS, 2015; Monteiro et al., 2018). El objetivo de esta revisión es dar a conocer el estado de la información del consumo de antioxidantes sintéticos en alimentos ultraprocesados, basados en los productos de la canasta básica mexicana.

\section{Material y métodos}

De febrero a junio del 2021, se realizó una búsqueda en inglés y español de publicaciones científicas relacionadas con las palabras clave: antioxidantes sintéticos, alimentos procesados, salud, toxicidad y genotoxicidad; en las bases de datos LILACS, PubMed y Google Académico. Los criterios de inclusión de las 
publicaciones científicas fueron: estudios originales de los últimos diez años, publicados en revistas con revisión por pares y sin restricción de idioma. Los criterios de exclusión fueron: estudios anteriores a los últimos diez ańos, estudios no respaldados por validez científica.

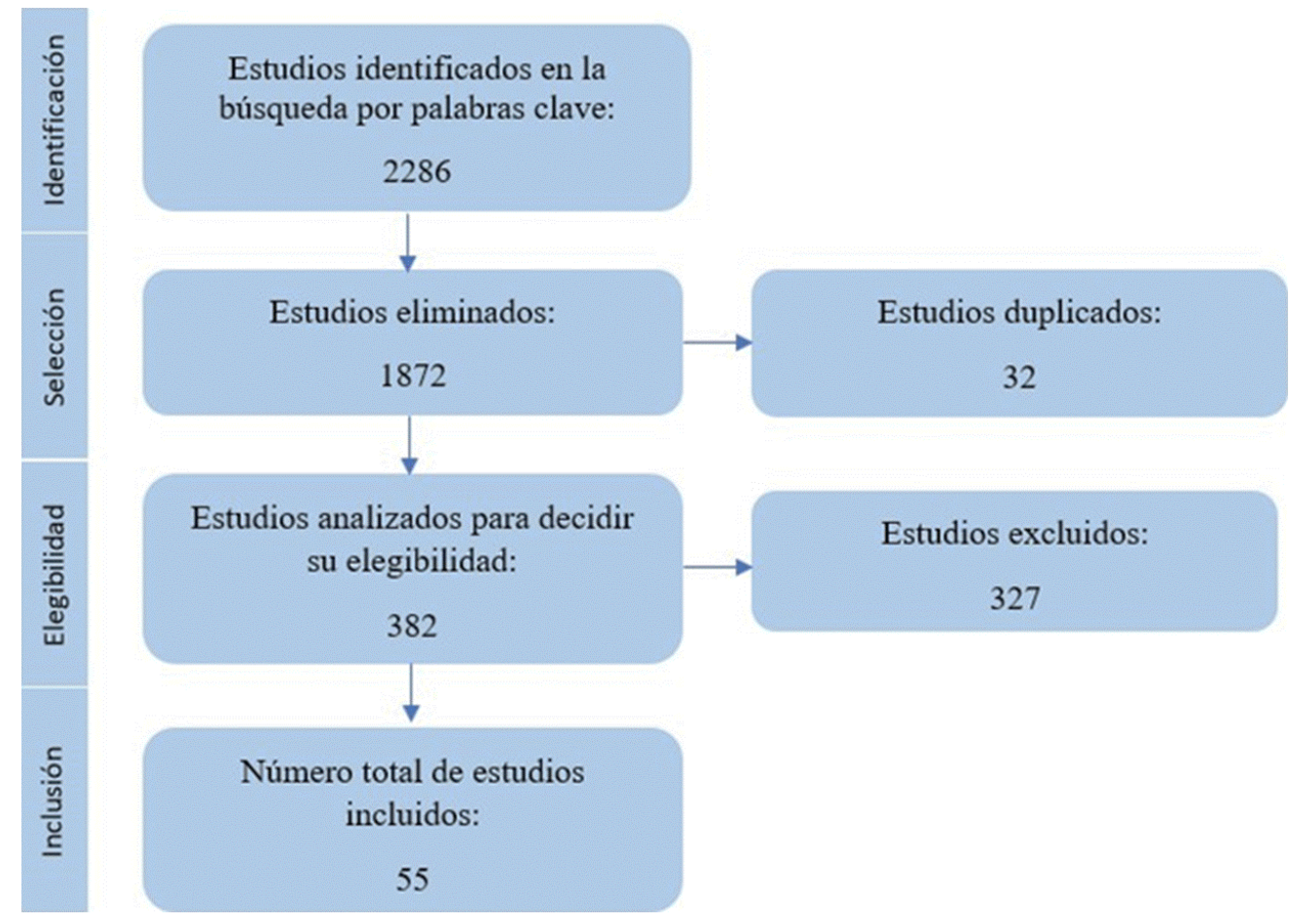

Figura 1. Metodología para inclusión de trabajos.

Fuente: elaboración propia.

Se seleccionaron alimentos representativos (con mayor presencia en los comercios, disponibles envasados o enlatados), comprendidos en la canasta básica mexicana, de tiendas de conveniencia y misceláneas representativas de los 125 municipios del estado de México, debido a mayor población demográfica (INEGI, 2020), se registró el contenido y la concentración de los antioxidantes BHA, BHT y TBHQ que se encuentra en la etiqueta de información nutricional del producto alimenticio. 
Los alimentos envasados o empaquetados fueron clasificados de acuerdo con el sistema NOVA, se reunieron los datos en 3 grupos: ingredientes culinarios procesados (NOVA 2), alimentos procesados (NOVA 3) y PUP (NOVA 4). Los alimentos sin procesar o mínimamente procesados (NOVA 1), no se registraron ya que carecen de etiqueta de información nutricional y por su definición no son agregados antioxidantes a esta clasificación.

\section{Resultados}

Entre los 40 productos que contempla la canasta básica mexicana, 33 son alimentos, los cuales incluyen aceite vegetal comestible, arroz, avena, atún, carne de pollo, carne de puerco, carne de res, chiles envasados, chocolate, concentrados sin azúcar para elaboración de bebidas, frijol, cuadro básico de frutas y verduras, frutas deshidratadas, galletas marías, de animales o saladas, garbanzos chicharos, soya, gelatina, golosina de amaranto o cacahuate, harina de maíz enriquecida, harina de trigo, huevo fresco, jamaica natural, leche fluida, en polvo y derivados de la leche, lentejas, maíz, pan de caja y de dulce, pasta para sopa, pescado seco, puré de tomate envasado, sal de mesa, sardina y tostadas. Con base en estos productos, se encontraron 53 que son comercializados en presentación de envase o lata de los cuales, el $71 \%$ menciona el antioxidante utilizado y solo el $15 \%$ su concentración. Basado en el sistema NOVA 39 productos (73\%) corresponden a los PUP, de los cuales once no mencionan el AS utilizado y ninguno la concentración; diez productos $(18 \%)$ corresponden a ingredientes culinarios procesados, donde el cien por ciento menciona el AS utilizado y solo ocho la concentración exacta; únicamente cuatro productos $(7 \%)$ corresponden a alimentos procesados y ninguno menciona el AS utilizado (Tabla 2). 
Tabla 2.

Clasificación NOVA e información de los antioxidantes presentes obtenidos de la etiqueta de información nutricional de los alimentos derivados de la canasta básica mexicana

\begin{tabular}{|c|c|c|c|c|c|}
\hline $\begin{array}{l}\text { Clasificación } \\
\text { NOVA }\end{array}$ & Producto & Tipo & Marca comercial & $\begin{array}{c}\text { Antioxidante que } \\
\text { presenta }\end{array}$ & Concentración \\
\hline \multirow{10}{*}{$\begin{array}{l}\text { NOVA } 2 \\
\text { Ingredientes } \\
\text { culinarios } \\
\text { procesados }\end{array}$} & \multirow{10}{*}{ Aceite vegetal } & Aceite de canola o girasol & $* 1-2-3^{\circledast}$ & TBHQ & $0.007 \%$ \\
\hline & & Puro de soya & ${ }^{*}$ Nutrioli $^{\oplus}$ & TBHQ & $0.01 \%$ \\
\hline & & Puro de maíz & ${ }^{*}$ La Gloria ${ }^{\otimes}$ & TBHQ & $0.008 \%$ \\
\hline & & Puro de maíz & Mazola $^{\oplus}$ & TBHQ & $0.01 \%$ \\
\hline & & $\begin{array}{l}\text { Aceite vegetal de soya, aceite vegetal } \\
\text { de canola }\end{array}$ & $\mathrm{Ave}^{\oplus}$ & TBHQ & $0.005 \%$ \\
\hline & & Puro de soya & Great value & TBHQ & $0.01 \%$ \\
\hline & & $\begin{array}{l}\text { Contiene } 2 \text { o más: soya, canola, } \\
\text { girasol }\end{array}$ & Aurrera ${ }^{\oplus}$ & TBHQ & $0.01 \%$ \\
\hline & & Aceite de canola & Capullo ${ }^{\circ}$ & TBHQ & $0.004 \%$ \\
\hline & & $\begin{array}{l}\text { Contiene } 2 \text { o más: canola, soja, } \\
\text { girasol, cártamo }\end{array}$ & Cristal $^{\oplus}$ & TBHQ & No mayor a $0.02 \%$ \\
\hline & & Puro de canola & Canoil $^{\oplus}$ & TBHQ & No mayor a $0.02 \%$ \\
\hline \multirow{4}{*}{$\begin{array}{l}\text { NOVA } 3 \\
\text { Alimentos } \\
\text { procesados }\end{array}$} & \multirow{3}{*}{ Atún } & Atún en aceite & Dolores ${ }^{\circledast}$ & No especificado & No especificado \\
\hline & & Atún en aceite & Tuny $^{\oplus}$ & No especificado & No especificado \\
\hline & & Atún en aceite & Herdez $^{\circledast}$ & No especificado & No especificado \\
\hline & Frijol & Frijoles bayos refritos & La Sierra ${ }^{\oplus}$ & No especificado & No especificado \\
\hline \multirow{11}{*}{$\begin{array}{l}\text { NOVA } 4 \\
\text { Alimentos } \\
\text { ultra - procesados }\end{array}$} & \multirow{2}{*}{ Avena } & Instantánea & $\mathrm{N} 1^{\oplus}$ & No especificado & No especificado \\
\hline & & Nucita patitas & Nutresa $^{\circledR}$ & TBHQ & No especificado \\
\hline & \multirow{2}{*}{ Chocolate } & Cremino & Nutresa $^{\oplus}$ & BHT у TBHQ & No especificado \\
\hline & & Crema de avellanas & Nutella ${ }^{\oplus}$ & No especificado & No especificado \\
\hline & \multirow[t]{3}{*}{ Frijol } & Frijoles negros refritos & Isadora ${ }^{\circledast}$ & $\mathrm{BHA}$ & No especificado \\
\hline & & Habaneras integrales & Gamesa ${ }^{\oplus}$ & TBHQ y BHT & No especificado \\
\hline & & Arcoiris & Gamesa $^{\oplus}$ & TBHQ & No especificado \\
\hline & \multirow{4}{*}{ Galletas } & Chocolatines & Gamesa $^{\oplus}$ & TBHQ y BHT & No especificado \\
\hline & & Emperador & Gamesa $^{\circledast}$ & TBHQ & No especificado \\
\hline & & **Marías & Gamesa $^{\oplus}$ & TBHQ & No especificado \\
\hline & & Cremax & Gamesa $^{\oplus}$ & TBHQ & No especificado \\
\hline
\end{tabular}


ESTADO DE LA INFORMACIÓN DEL CONSUMO EN MÉXICO DE ANTIOXIDANTES SINTÉTICOS EN ALIMENTOS ULTRA-PROCESADOS, BASADOS EN LOS PRODUCTOS DE LA CANASTA BÁSICA Carbajal-SÁnchez, Ramírez-Durán, Gamboa-Angulo, Moreno-PÉrez

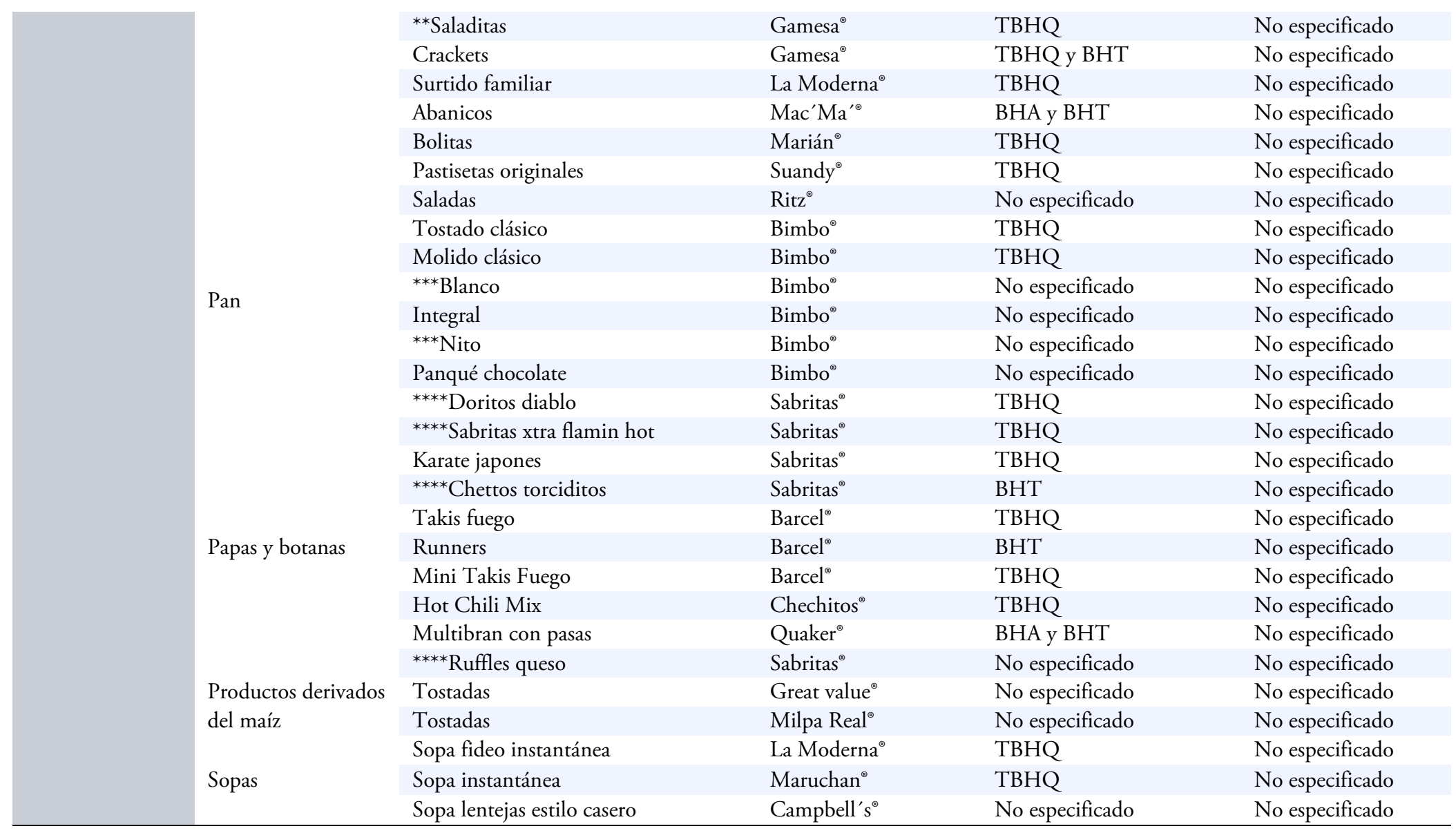

Fuente: elaboración propia.*Aceites más consumidos en México ${ }^{*}$ Seales \& Associates, 2017. ${ }^{* *}$ Galletas más consumidas en México **legiscomex, 2012. ${ }^{* * *}$ Pan más consumido en México ${ }^{* * *}$ BIMBO, 2019. Papas más consumidas en México. ${ }^{* * * *}$ López, 2018. ${ }^{* * * *}$ 


\section{Discusión}

De acuerdo con los resultados, los 53 productos alimenticios derivados de la canasta básica mexicana se clasifican dentro de alguno de los grupos NOVA; es el grupo NOVA 4 (PUP) el que tuvo mayor representatividad, este resultado coincide con lo reportado por Barquera y Rivera (2020), quienes mencionan que la dieta de los mexicanos ha cambiado de productos frescos y sin procesar a PUP. Los PUP son formulaciones elaboradas industrialmente a partir de sustancias extraídas o derivadas de alimentos con aditivos agregados, éstos alimentos incluyen refrescos, bebidas, jugos, bocadillos dulces y salados, confitería, pan industrializado, pastelitos y galletas, cereales endulzados, productos cárnicos reconstituidos, margarina, mayonesa, papas fritas, chocolate, helado, platos de pasta y salsas instantáneas, entre otros (OMS, 2015; OPS 2019). México es el mayor consumidor de PUP en América Latina y el cuarto a nivel mundial, notoriamente, la población preescolar es quien más los consume, donde cerca del $40 \%$ de sus calorías provienen de la ingestión de estos productos (UNICEF, 2020).

Respecto al contenido de la información del AS utilizado es notable que casi el $30 \%$ del total de los productos que derivan de la canasta básica mexicana no mencionan el tipo de AS utilizado y más del $81 \%$ no mencionan la concentración. En México la Secretaría de Salud a través de la Comisión Federal para la Protección contra Riesgos Sanitarios (Cofepris) es la que aprueba todos los aditivos usados en México y realiza las acciones para la vigilancia sanitaria a nivel nacional (Secretaría de Salud, 2012). Las normas mexicanas NOM-218-SSA1-2011, NOM186SSA1/SCFI-2013 y NOM-213-SSA1-2018 se basan en el "Acuerdo por el que se determinan los aditivos y coadyuvantes en alimentos, bebidas y suplementos alimenticios, su uso y disposiciones sanitarias" donde se establece el límite máximo en miligramos por kilogramo o litro, en que puede ser utilizado un aditivo de acuerdo con el alimento en el que se adicione. La falta de información precisa sobre el contenido y concentración de los antioxidantes en las etiquetas de información nutricional en México no cumplen con este acuerdo, el cual menciona que los aditivos deberán indicarse en la declaración de ingredientes contenida en el etiquetado. 
La exposición dietética se define como la "ingesta total de una sustancia química por los seres humanos" (WHO, 1987) y esta se puede determinar: a) mediante la presencia cuantitativa de un aditivo en alimentos y dietas individuales; b) los patrones de ingesta de los alimentos individuales que contienen los aditivos alimentarios pertinentes y c) la posibilidad de que los consumidores que consumen grandes cantidades de los alimentos dados y el aditivo alimentario relevante esté presente en estos alimentos en concentraciones mayores a las recomendadas (Jain y Mathur, 2015). La estimación de la exposición alimentaria resultante para una población puede compararse con el valor de referencia basado en guías de salud (WHO, 2020), sin embargo, debido a la diversidad de aditivos, estimar la exposición de la población puede ser complicado y costoso (Jain y Mathur, 2015). En México se requiere información precisa de los alimentos que son fuentes potenciales de BHA, BHT y TBHQ en cada región metropolitana, así como su ingestión media diaria per cápita nacional para poder estimar la ingesta diaria máxima teórica, como en el caso de Brasil (Maziero, Baunwart y Toledo, 2010).

Por otra parte, el Comité Mixto FAO/OMS de Expertos en Aditivos Alimentarios (JECFA) evalúa los aditivos alimentarios basado en datos bioquímicos y toxicológicos para determinar si un aditivo alimentario puede ser utilizado sin perjuicio a la salud, para ello se determina la "Ingestión Diaria Admisible" (IDA), que es la cantidad segura estimada en $\mathrm{mg} / \mathrm{kg}$ peso corporal que puede consumir una persona de una sustancia presente en alimentos o bebidas y que no causa un dańo apreciable a la salud. Esto se determina mediante los estudios de toxicidad aguda, a corto y a largo plazo, observándose el comportamiento de la sustancia en el organismo como la absorción, distribución y excreción (Carrington y Bolger, 2014). Los AS encontrados en los productos envasados o enlatados derivados de la canasta básica mexicana son el BHA, BHT y TBHQ, que son comúnmente utilizados en grasas, aceites y sus derivados, aunque también pueden ser utilizados en el envasado de los alimentos para prevenir su degradación durante el procesamiento y almacenamiento (Fasihnia et al., 2020); sin embargo, elevadas concentraciones de antioxidantes exógenos pueden alterar la homeostasis redox en el organismo (Bouayed and Bohn, 2010). Estos antioxidantes son denominados sintéticos ya que son creados en laboratorios, la mayoría a partir de la molécula del benceno $(1,3,5-$ ciclohexatrieno, $\left.\mathrm{C}_{6} \mathrm{H}_{6}\right)$, que es un hidrocarburo aromático derivado de la destilación del petróleo (Pulgarín, 2016). En México, se encuentra una iniciativa para reformar el artículo 282 de la Ley General de Salud, que pretende prohibir el uso de BHA y 
BHT como aditivos en alimentos (Armenta, 2020), sin embargo aún no ha sido aprobada. Una alternativa sería promover el uso de antioxidantes de origen natural para sustituir a los antioxidantes fenólicos sintéticos.

Respecto a la concentración del AS utilizada, es preocupante que la mayoría de los productos envasados y enlatados no lo especifique. La Secretaria de Salud regula los límites máximos de cada antioxidante en México, de acuerdo con la IDA establecida en el Codex Alimentarius: BHA ( $40400 \mathrm{mg} / \mathrm{kg}$ o $1000 \mathrm{mg} / \mathrm{L}$ ), BHT (24$400 \mathrm{mg} / \mathrm{kg}$ o $1000 \mathrm{mg} / \mathrm{L}$ ) y TBHQ (100-200 mg/kg) (Cofepris y Secretaría de Salud, 2021; Secretaría de Salud, 2012), los cuales se encuentran dentro de los intervalos recomendados por el Codex Alimentarius: BHA (100 - $400 \mathrm{mg} / \mathrm{kg})$, BHT (75-400 mg/kg) y TBHQ (100-400 mg/kg) (FAO/WHO, 2019).

No obstante, Wang y Kannan, (2019), mencionan que además de la exposición de estos antioxidantes fenólicos sintéticos en alimentos, se debería considerar la exposición a éstos aditivos a través de otras fuentes de exposición, como la inhalación de polvo o en el uso de cosméticos que los contengan. No se encontró información de la exposición cualitativa o cuantitativa de AS en la población mexicana, contrariamente a países como Alemania donde se investigó la exposición al BHT en niños y adolescentes (Murawski et al., 2021); en población general de Arabia Saudita, China, Estados Unidos, India y Japón (Wang y Kannan, 2019); en Francia por medio de los datos de consumo individual combinados con los niveles máximos permitidos se estimó en niños menores de 3 años la exposición a BHA y BHT (Mancini et al., 2015). Es notorio que en Latinoamérica solo se han encontraron estudios de Brasil, donde mediante datos de consumo de alimentos, derivados de una encuesta en hogares y productos envasados, se estimaron las ingestas diarias máximas teóricas de BHA, BHT y TBHQ (Maziero et al., 2010).

Basado en la evidencia de la toxicidad de los AS BHA, BHT y TBHQ, es importante estimar la exposición a estos aditivos alimentarios, ya que existe la posibilidad de que la población consuma estos antioxidantes por encima de la concentración recomendada debido a sus hábitos de alimentación. Como ejemplo $>10 \%$ de grasa saturada de la ingestión energética diaria total recomendada por la OMS, (FAO, 2010), esta situación, podría generar el riesgo de consumir más AS agregados a los aceites para evitar su oxidación o cuando por facilidad de preparación se prefieren los alimentos procesados y PUP (Nieto-Orozco et al., 2017). 
México es el país que consume la mayor cantidad de PUP per cápita, así como el que presenta las mayores ventas de estos productos en América Latina (Matos, Adams y Sabaté, 2021). Ya que a la mayoría de PUP se les agregan AS, su ingestión puede ser subestimada. La ingestión real de antioxidantes es poco conocida ya que existe falta de información sobre la exposición humana a estos compuestos (Tortosa et al. 2020). La carencia de la información real de la cantidad de AS adicionados a los alimentos que derivan de la canasta básica mexicana, así como la concentración, provoca que estimar la IDA en la población mexicana sea un proceso incierto.

\section{Conclusiones}

De acuerdo con el sistema NOVA, la mayor proporción de los productos derivados de la canasta básica mexicana se encuentran dentro de la clasificación de PUP. Solo la menor proporción de los alimentos PUP menciona la concentración de AS. Más de la mitad de los productos disponibles en el mercado no cumplen con las regulaciones mexicanas sobre la información del etiquetado. Debido a la falta de información del contenido y concentración de los AS en las etiquetas nutricionales, es probable que la población mexicana consuma más BHA, BHT y TBHQ que la recomendada por la Secretaría de Salud. La IDA puede ser subestimada, por lo que es necesario realizar más investigaciones sobre la exposición dietética a AS en los mexicanos.

\section{Referencias}

Abo-EL-Sooud, K., Hashem, M. M., Badr, Y. A., Eleiwa, M. M. E., Gab-Allaha, A. Q., Abd-Elhakim, Y. M. y BahyEL-Dien, A. (2018). Assessment of hepato-renal damage and genotoxicity induced by long-term exposure to five permitted food additives in rats. Environmental Science and Pollution Research, 25(26). doi: https://doi.org/10.1007/ s11356-018-2665-z 
Alofe, O., Kisanga, E., Inayat-hussain, S. H., Fukumura, M., Garcia-milian, R., Perera, L., Vasiliou, V. y Whirledge, S. (2019). Determining the endocrine disruption potential of industrial chemicals using an integrative approach: Public databases, in vitro exposure, and modeling receptor interactions. Environment International, 131(104969). doi: https://doi.org/10.1016/j.envint.2019.104969

Armenta, A. (2020). Iniciativa con proyecto de decreto por el que se reforma. México: Senado de la República. 1-112.

Atta, E. M., Mohamed, N. H. y Abdelgawad, A. A. M. (2017). Antioxidants: An Overview on the Natural and Synthetic Types. European Chemical Bulletin, 6(8), 365. doi: https://doi.org/10.17628/ecb.2017.6.365-375

Baran, A., Yildirim, S., Ghosigharehaghaji, A., Bolat, I., Sulukan, E. y Ceyhun, S. (2020). An approach to evaluating the potential teratogenic and neurotoxic mechanism of BHA based on apoptosis induced by oxidative stress in zebrafish embryo (Danio rerio). Human and Experimental Toxicology, XX(X)1-14. doi: https://doi.org/10.1177/ 0960327120952140

Barquera, S. y Rivera, J. A. (2020). Obesity in Mexico: rapid epidemiological transition and food industry interference in health policies. The Lancet Diabetes and Endocrinology, 8(9), 746-747. doi: https://doi.org/10.1016/S2213-8587(20)30269-2

BIMBO (2019). Reporte de Resultados 2019. Recuperado de https://www.grupobimbo. com/es/grupo-bimbo-reporteresultados-2019

Bouayed, J. y Bohn, T. (2010). Exogenous antioxidants -Double-edged swords in cellular redox state Health beneficial effects at physiologic doses versus deleterious effects at high doses. Oxidative Medicine and Cellular Longevity, 3(4), 228-237. doi: https://doi.org/10.4161/oxim.3.4.12858

Carrington, C. D. y Bolger, P. M. (2014). Food Safety and Toxicology. Encyclopedia of Toxicology. Third Edition (pp. 639-644). Elsevier. doi: https://doi.org/10.1016/B9780-12-386454-3.00022-1

Comisión Federal para la Protección contra Riesgos Sanitarios y Secretaría de Salud (Cofepris y SS, 2021) ANEXO I Aditivos con diversas clases funcionales y con una IDA establecida. México.

Domínguez, R., Pateiro, M., Gagaoua, M., Barba, F. J., Zhang, W. y Lorenzo, J. M. (2019). A comprehensive review on lipid oxidation in meat and meat products. Antioxidants, 8(429), 1-31. doi: https://doi.org/10.3390/antiox8100429

Eskandani, M., Hamishehkar, H., Ezzati, J. y Dolatabadi, N. (2014). Cytotoxicity and DNA damage properties of tert-butylhydroquinone (TBHQ) food additive. Food Chemistry, 153(2014), 315-320. doi: https://doi.org/10.1016/j.foodchem.2013.12.087 
ESTADO DE LA INFORMACIÓN DEL CONSUMO EN MÉXICO DE ANTIOXIDANTES SINTÉTICOS

EN ALIMENTOS ULTRA-PROCESADOS, BASADOS EN LOS PRODUCTOS DE LA CANASTA BÁSICA

Organización de las Naciones Unidas para la Alimentación y la Agricultura y Organización Mundial de la Salud (FAO/WHO, 2019). General standard for food additives. Codex Alimentarius, 1-480. Recuperado de https://www.fao.org/fao-who-codexalimentarius/ sh-proxy/en/?lnk=1 \&url=https\%253A\%252F\%252Fworkspace.fao.org\%252Fsites \%252Fcodex\%252FStandards\%252FCXS\%2B192-1995\%252FCXS_192e.pdf

FAO (2010). Fats and fatty acids in human nutrition: Introduction. Annals of Nutrition and Metabolism, 55. doi: https://doi.org/10.1159/000228993

Fasihnia, S. H., Peighambardoust, S. H., Peighambardoust, S. J., Oromiehie, A., Soltanzadeh, M. y Peressini, D. (2020). Migration analysis, antioxidant, and mechanical characterization of polypropylene-based active food packaging films loaded with BHA, BHT, and TBHQ. Journal of Food Science, 85(8), 1-12. doi: https://doi.org/10.1111/1750-3841.15337

Halliwell, B. (1995). The definition and measurement of antioxidants. Free Radical Biology and Medicine, 18(I), 125-126.

Ham, J., Lim, W., Whang, K. y Song, G. (2019). Butylated hydroxytoluene induces dysregulation of calcium homeostasis and endoplasmic reticulum stress resulting in mouse. Environmental Pollution, 256. doi: https://doi.org/10.1016/j.envpol.2019. 113421

Ham, J., Lim, W., You, S. y Song, G. (2019). Butylated hydroxyanisole induces testicular dysfunction in mouse testis cells by dysregulating calcium homeostasis and stimulating endoplasmic reticulum stress. Science of the Total Environment, 702(2020), 134775. doi: https://doi.org/10.1016/j.scitotenv.2019.134775

Instituto Nacional de Estadística y Geografía (INEGI, 2020). Presentación de resultados censo 2020. Recuperado de https:/www.inegi.org.mx/contenidos/programas/ccpv/ 2020/doc/Censo2020_Principales_resultados_ejecuti va_EUM.pdf

Jain, A. y Mathur, P. (2015). Estimation of Food Additive Intake-Overview of the Methodology. Food Reviews International, 31, 355-384. doi: https://doi.org/10.1080/ 87559129.2015.1022830

Karimi, Z., Ezzati, J., Dolatabadi, N. y Dehghan, P. (2019). The protective effect of thymoquinone on tertbutylhydroquinone induced cytotoxicity in human umbilical vein endothelial cells. Toxicology Research, 1050-1056. doi: https://doi.org/10.1039/ c9tx00235a

Legiscomex. (2012). Botanas, un negocio que va en crecimiento. Recuperado de https://www. legiscomex.com/Documentos/botanas-mexico-virginia-perez-actualizacion

Liang, X., Zhao, Y., Liu, W., Li, Z., Souders, C. L. y Martyniuk, C. J. (2019). Butylated hydroxytoluene induces hyperactivity and alters dopamine-related gene expression in larval zebrafish (Danio rerio). Environmental Pollution, 113624. doi: https://doi.org/10.1016/j.envpol.2019.113624 
López, O. (2018). Cultura organizacional y productividad. Estudio de caso en una microempresa productora de botanas en Metepec, Estado de México, 2018. (Tesis de pregrado). Recuperado de: http://ri.uaemex.mx/bitstream/handle/20.500.11799/ 95193/TESIS\%20Omar\%20L\%C3\%B3pez.pd? sequence=1 \&isAllowed $=y$

Lourenço, S. C., Moldão-Martins, M. y Alves, V. D. (2019). Antioxidants of natural plant origins: From sources to food industry applications. Molecules, 24(4132), 1-25. doi: https://doi.org/10.3390/molecules24224132

Mancini, F. R., Paul, D., Gauvreau, J., Volatier, J. L., Vin, K. y Hulin, M. (2015). Dietary exposure to benzoates (E210E213), parabens (E214-E219), nitrites (E249-E250), nitrates (E251-E252), BHA (E320), BHT (E321) and aspartame (E951) in children less than 3 years old in France. Food Additives and Contaminants-Part A, 32(3), 293306. doi: https://doi.org/10.1080/19440049.2015.1007535

Martínez, S. (2000). La Canasta Básica Alimentaria en México, 1980-1998: Contenido y Determinantes. 157. Recuperado de http://www.economia.unam.mx/secss/docs/tesisfe/ MartinezRSE/Tesis.pdf

Matos, R. A., Adams, M. y Sabaté, J. (2021). Review: The Consumption of UltraProcessed Foods and Non-communicable Diseases in Latin America. Frontiers in Nutrition, 8. doi: https://doi.org/10.3389/fnut.2021.622714

Maziero, G. C., Baunwart, C. y Toledo, M. C. F. (2010). Estimates of the theoretical maximum daily intake of phenolic antioxidants BHA, BHT and TBHQ in Brazil. Food Additives and Contaminants, 18(5), 37-41. doi: https://doi.org/10.1080/0265203012 0645

Monteiro, C. A., Cannon, G., Levy, R. B., Moubarac, J. C., Louzada, M. L. C., Rauber, F., Khandpur, N., Cediel, G., Neri, D., Martinez-Steele, E., Baraldi, L. y Jaime, P. C. (2019). Ultra-processed foods: What they are and how to identify them. Public Health Nutrition, 22(5), 936-941. doi: https://doi.org/10.1017/S1368980018003762

Murawski, A., Schmied-Tobies, M., Rucic, E., Schmidtkunz, C., Küpper, K., Leng, G., Eckert, E., Kuhlmann, L., Göen, T., Daniels, A., Schwedler, G. y Kolossa-Gehring, M. (2021). Metabolites of 4-methylbenzylidene camphor (4-MBC), butylated hydroxytoluene (BHT), and tris(2-ethylhexyl) trimellitate (TOTM) in urine of children and adolescents in Germany-human biomonitoring results of the German Environmental Survey GerES V (2014-2017). Environmental Research, 192(2021), 110345.

Nieto-Orozco, C., Chanin Sangochian, A., Tamborrel Signoret, N., Vidal-González, E., Tolentino-Mayo, L. and Vergara-Castańeda, A. (2017). Percepción sobre el consumo de alimentos procesados y productos ultraprocesados en estudiantes de posgrado de la Ciudad de México. Journal of Behavior, Health \& Social Issues, 9(2), 82-88. doi: https://doi.org/10.1016/j.jbhsi.2018.01.006 
ESTADO DE LA INFORMACIÓN DEL CONSUMO EN MÉXICO DE ANTIOXIDANTES SINTÉTICOS

EN ALIMENTOS ULTRA-PROCESADOS, BASADOS EN LOS PRODUCTOS DE LA CANASTA BÁSICA

Organización Panamericana de la Salud y Organización Mundial de la Salud (OPS y OMS, 2015). Alimentos y bebidas ultraprocesados en América Latina. Recuperado de http://iris.paho.org/xmlui/bitstream/handle/123456789/7698/9789275318645_esp.p $\mathrm{df}$ ? sequence $=5$

OPS (2019). Alimentos y bebidas ultraprocesados en América Latina: ventas, fuentes, perfiles de nutrientes e implicaciones normativas. Recuperado de https://iris.paho.org/ bitstream/handle/10665.2/51523/9789275320327_spa.pdf?sequence=1 1 \&isAllowed=y

Park, S., Lee, J., Lim, W., You, S. y Song, G. (2019). Butylated Hydroxyanisole Exerts Neurotoxic E ff ects by Promoting Cytosolic Calcium Accumulation and Endoplasmic Reticulum Stress in Astrocytes. J. Agric. Food Chem. 67, 9618-9629. doi: https://doi.org/10.1021/acs.jafc.9b02899

Pulgarín, J. F. (2016). Una didáctica para el estudio del benceno en productos de consumo masivo: aprendizaje con participación ciudadana. (Tesis de maestría). Medillín: Universidad de Antioquia.

Salehi, B., Martorell, M., Arbiser, J. L., Sureda, A., Martins, N., Maurya, P. K., SharifiRad, M., Kumar, P. y Sharifi-Rad, J. (2018). Antioxidants: Positive or Negative Actors? Biomolecules, 8(4). doi: https://doi.org/10.3390/biom8040124

Secretaría de Salud (SS, 2012) Acuerdo por el que se determinan los aditivos y coadyuvantes en alimentos, bebidas y suplementos alimenticios, su uso y disposiciones sanitarias. Diario Oficial de la Federación. Recuperado de https://dof.gob.mx/nota_detalle_popup.php? codigo $=5259470$

Seales and Associates (2017). Reporte de la Industria del Aceite de Consumo. 9. Recuperado de http://mnamexico.com/wp-content/uploads/2017/08/Aceite-compressed.pdf

Shahidi, F. y Abad, A. (2018). Lipid-derived flavours and off-flavours in food. Encyclopedia of Food Chemistry, Elsevier. doi: https://doi.org/10.1016/B978-0-08-100596-5.216661

Shankar, S., Gowthaman, N. S. K., Arul, P., Chen, F., Lim, H. N. y Qin, F. X. (2020). Ultra-sensitive and selective determination of a phenolic food additive using protein capped gold nanoclusters: a dual in-line fluorometric and colorimetric sensing probe. New Journal of Chemistry, 45(3), 1278-1285. doi: https://doi.org/10.1039/d0nj04712k

Sugiharto, S. (2019). A review of filamentous fungi in broiler production. Annals of Agricultural Sciences. Faculty of Agriculture, Ain-Shams University. doi: https://doi.org/10.1016/j.aoas.2019.05.005

Sun, Z., Tang, Z., Yang, X., Liu, Q. S., Liang, Y., Fiedler, H. y Zhang, J. (2019). Perturbation of 3-tert-butyl-4hydroxyanisole in adipogenesis of male mice with normal and high fat diets. Science of the Total Environment, 703(10), 135608. doi: https://doi.org/10.1016/j.scitotenv.2019.135608 
Sun, Z., Yang, X., Liu, Q. S., Li, C., Zhou, Q., Fiedler, H., Liao, C., Zhang, J. and Jiang, G. (2019). Butylated hydroxyanisole isomers induce distinct adipogenesis in 3T3-L1 cells. Journal of Hazardous Materials, 379. doi: https://doi.org/10.1016/j.jhazmat. 2019.120794

Tortosa, V., Pietropaolo, V., Brandi, V., Macari, G., Pasquadibisceglie, A. y Polticelli, F. (2020). Computational Methods for the Identification of Molecular Targets of Toxic Food Additives. Butylated Hydroxytoluene as a Case Study. Molecules, 25, 1-17.

Fondo de las Naciones Unidas para la Infancia (UNICEF, 2020). ¿Qué estamos esperando? Recuperado de https://www.unicef.org/es/historias/que-estamos-esperando-obesidadinfantil-mexico

Vandghanooni, S., Forouharmehr, A., Eskandani, M., Barzegari, A., Kafil, V., Kashanian, S. y Ezzati Nazhad Dolatabadi, J. (2013). Cytotoxicity and DNA fragmentation properties of butylated hydroxyanisole. DNA and Cell Biology, 32(3), 98-103. doi: https://doi.org/10.1089/dna.2012.1946

Wang, W. y Kannan, K. (2019). Quantitative identification of and exposure to synthetic phenolic antioxidants, including butylated hydroxytoluene, in urine. Environment International , 128, 24-29. doi: https://doi.org/10.1016/j.envint.2019.04.028

Organización Mundial de la Salud (WHO, 1987). Principles for the safety assessment of Food Additives and Contaminants in food. Recuperado de https://apps.who.int/iris/ bitstream/handle/10665/37578/9241542705eng.pdf?sequence $=1 \&$ isAllowed $=\mathrm{y}$

WHO (2020). Dietary Exposure Assessment of Chemicals in Food. Recuperado de https://www.who.int/docs/defaultsource/chemical-safety/ehc240-chapter6-edited(41).pdf?sfvrsn=96810319_0

Xu, X., Liu, A., Hu, S., Ares, I., Martínez-Larrañaga, M. R., Wang, X., Martínez, M., Anadón, A. y Martínez, M. A. (2021). Synthetic phenolic antioxidants: Metabolism, hazards and mechanism of action. Food Chemistry, 353. doi: https://doi.org/10.1016/ j.foodchem.2021.129488

Yang, X., Sun, Z., Wang, W., Zhou, Q. y Shi, G. (2018). Developmental toxicity of synthetic phenolic antioxidants to the early life stage of zebrafis. Science of the Total Environment, 643, 559-568. doi: https://doi.org/10.1016/j.scitotenv.2018.06.213

Zhang, R., Li, J. y Cui, X. (2020). Science of the Total Environment Tissue distribution , excretion, and metabolism of 2, 6-di- tert -butyl-hydroxytoluene in mice. Science of the Total Environment, 739, 139862. doi: https://doi.org/10.1016/j.scitotenv.2020.1398 62 
Zhao, H., Xu, J., Yan, Z., Ren, H. y Zhang, Y. (2020). Microplastics enhance the developmental toxicity of synthetic phenolic antioxidants by disturbing the thyroid function and metabolism in developing zebra fi sh. Environment International, 140. doi: https://doi.org/10.1016/j.envint.2020.105750

Zhao, Y., Zhao, W., Liu, L. y Wang, Y. (2020). TBHQ-Overview of multiple mechanisms against oxidative stress for attenuating methamphetamine-induced neurotoxicity. Oxidative Medicine and Cellular Longevity . doi: https://doi.org/10.1155/2020/887 4304 